\title{
COMPRESSIBLE MAPS
}

\author{
JAY E. GOLDFEATHER
}

\begin{abstract}
Weingram has shown that if $G$ is a finitely generated abelian group, then every nontrivial map $f: \Omega S^{2 n+1} \rightarrow K(G, 2 n)$ is incompressible; that is, $f$ is not homotopic to a map whose image is contained in some finitedimensional skeleton.

It is shown that a nontrivial map $\Omega S^{2 n+1} \rightarrow K(G, 2 n)$ may be compressible if $G$ is not finitely generated. This result leads to some understanding of the obstructions to compressibility in Weingram's Theorem.
\end{abstract}

A map $f: X \rightarrow Y$ is said to compress into $A \subseteq Y$ if there is a map $f^{\prime}: X \rightarrow A$ such that $f \simeq i \cdot f^{\prime}$, where $i$ is inclusion. Weingram [4] has shown that if $G$ is a finitely generated abelian group, then every nontrivial map $f: \Omega S^{2 n+1} \rightarrow K(G, 2 n)$ is incompressible, that is, $f$ is not homotopic to a map whose image is contained in some finite-dimensional skeleton.

It will be shown that a nontrivial map $\Omega S^{2 n+1} \rightarrow K(G, 2 n)$ may be compressible if $G$ is not finitely generated. Specifically, if $P$ is a set of primes and $Z_{P} \subseteq Q$ is the subgroup of $P$-local integers, then any map $\Omega S^{2 n+1}$ $\rightarrow K\left(Q / Z_{P}, 2 n\right)$ compresses into the $(2 n+1)$-skeleton, provided $2 \notin P$ or $n$ $=1,3$.

Let $M(G, k)$ denote a Moore space of type $(G, k)$. For the remainder of this paper, it will be assumed that $2 \notin P$ or $2 \in P$ and $n=1,3$.

The following two well-known theorems will be stated without proof:

TheOREM (ADAMS [1]). $M\left(Z_{P}, 2 n+1\right)$ is an $H$-space.

Theorem (Stasheff [3]). Let $X$ and $W$ be $H$-spaces and let $F: X \rightarrow W$ be an $H$-map. Let $\Omega W \rightarrow Y \rightarrow X$ be the fibration induced by $f$. Then $Y$ is an $H$-space.

Lemma 1. Any map $M\left(Z_{P}, 2 n+1\right) \rightarrow K(Q, 2 n+1)$ is homotopic to an $H$ map.

Proof. The obstructions to a map $f: X \rightarrow Y$ being homotopic to an $H$-map lie in $H^{i}\left(X \wedge X ; \pi_{i}(Y)\right)$. Since

$$
\pi_{i}(K(Q, 2 n+1))=0 \text { for } i \neq 2 n+1
$$

and

$$
H^{2 n+1}\left(M\left(Z_{P}, 2 n+1\right) \wedge M\left(Z_{P}, 2 n+1\right) ; G\right)=0 \text { for any } G,
$$

all obstructions lie in zero groups.

Received by the editors January 29, 1976 and, in revised form, March 22, 1976.

AMS (MOS) subject classifications (1970). Primary 55G36; Secondary 55F05, 55D45.

Key words and phrases. $H$-space $\bmod p$. 
In view of the preceding lemma, it will be assumed that any given map $f: M\left(Z_{P}, 2 n+1\right) \rightarrow K(Q, 2 n+1)$ is an $H$-map.

Let $f$ be induced by the natural embedding $Z_{P} \subseteq Q$.

Proposition 2. Let $K(Q, 2 n) \rightarrow E \rightarrow M\left(Z_{P}, 2 n+1\right)$ be the fibration induced by $f$. Then $E$ is an $H$-space.

Proof. This is an immediate consequence of the above two theorems and Lemma 1.

THEOREM 3.

$$
\tilde{H}_{i}(E ; Z)= \begin{cases}Q / Z_{P}, & i=2 n \\ 0, & \text { otherwise }\end{cases}
$$

Proof. (Porter [2] has proved a similar theorem when the base space is $S^{3}$.) $H_{*}(K(Q, 2 n) ; Z)$ is a polynomial algebra over $Z$ on one generator in dimension $2 n$ tensored with $Q$. Since $f$ is induced by the natural embedding of $Z_{P} \subseteq Q$, the Serre exact sequence

$$
H_{2 n+1}(E) \rightarrow H_{2 n+1}\left(M\left(Z_{P}, 2 n+1\right)\right) \rightarrow H_{2 n}(K(Q, 2 n)) \rightarrow H_{2 n}(E) \rightarrow 0
$$

reduces to

$$
0 \rightarrow Z_{P} \rightarrow Q \rightarrow H_{2 n}(E) \rightarrow 0
$$

and, hence, $H_{2 n}(E)=Q / Z_{P}$.

In the homology spectral sequence, the only nonzero differential is $d^{2 n+1}$ which is an isomorphism for total degrees greater than $2 n+1$ since $Z_{P} \otimes Q$ $\cong Q$. Hence, $H_{i}(E)=0$ for $i>2 n+1$.

Remark. $E$ is homotopic to a $(2 n+1)$-dimensional Moore space

$$
M\left(Q / Z_{P}, 2 n\right) .
$$

Proposition 4. $K\left(Q / Z_{P}, 2 n\right)$ is not homotopic to a finite-dimensional complex.

Proof. The exact sequence $0 \rightarrow Z_{P} \rightarrow Q \rightarrow Q / Z_{P} \rightarrow 0$ induces a fibration

$$
K\left(Q / Z_{P}, 2 n\right) \rightarrow K\left(Z_{P}, 2 n+1\right) \rightarrow K(Q, 2 n+1) .
$$

It is well known that $K(Q, 2 n+1)$ is homotopic to a finite-dimensional complex. Hence if $K\left(Q / Z_{P}, 2 n\right)$ were also homotopic to a finite-dimensional complex, it would imply that $H^{k}\left(K\left(Z_{P}, 2 n+1\right) ; Z_{p}\right)=0$ for all $k$ greater than some integer. It suffices to show, then, that $H^{k}\left(K\left(Z_{P}, 2 n+1\right) ; Z_{p}\right)$ is nonzero for infinitely many $k$, where $p \in P$.

Let $\iota$ be the fundamental class in $H^{2 n+1}\left(K(Z, 2 n+1) ; Z_{p}\right)=\operatorname{Hom}\left(Z, Z_{p}\right)$. It is well known that there are an infinite number of Steenrod operations $\mathscr{P} I$ such that $\mathscr{P}^{I} \iota \neq 0$. ( $I$ denotes an admissible sequence.) Hence it suffices to show that if $f: K(Z, 2 n+1) \rightarrow K\left(Z_{P}, 2 n+1\right)$ is the natural inclusion, then 
$f^{*}(\tau)=\imath$ for some $\imath \in H^{2 n+1}\left(K\left(Z_{P}, 2 n+1\right) ; Z_{p}\right)=\operatorname{Hom}\left(Z_{P}, Z_{p}\right)$. But $f^{*}$ : $\operatorname{Hom}\left(Z_{P}, Z_{p}\right) \rightarrow \operatorname{Hom}\left(Z, Z_{p}\right)$ is onto and the proposition is proved.

THEOREM 5. Every nontrivial map $\Omega S^{2 n+1} \rightarrow K\left(Q / Z_{P}, 2 n\right)$ compresses into the $(2 n+1)$-skeleton.

Proof. Any map $f: \Omega S^{2 n+1} \rightarrow K\left(Q / Z_{p}, 2 n\right)$ is homotopic to a map $r \cdot \Omega S g$, where $g: S^{2 n} \rightarrow K\left(Q / Z_{P}, 2 n\right)$ and $r$ is the retraction $\Omega S K\left(Q / Z_{P}, 2 n\right)$ $\rightarrow K\left(Q / Z_{P}, 2 n\right)$.

But every such map $g$ factors through $M\left(Q / Z_{P}, 2 n\right)$ so that

$$
S^{2 n} \stackrel{g_{1}}{\longrightarrow} M\left(Q / Z_{P}, 2 n\right) \stackrel{g_{2}}{\longrightarrow} K\left(Q / Z_{P}, 2 n\right)
$$

is nontrivial where

$$
g \simeq g_{2} \cdot g_{1}
$$

By Theorem 3 and Proposition $2, M\left(Q / Z_{P}, 2 n\right)$ is an $H$-space so that $g_{1}$ extends to $\bar{g}_{1}: \Omega S^{2 n+1} \rightarrow M\left(Q / Z_{P}, 2 n\right)$. Then $f \simeq g_{2} \cdot \bar{g}_{1}$, so $f$ compresses into the $(2 n+1)$-skeleton.

Observe that if $P=\{p\}$, then $Q / Z_{P}=Z_{p^{\infty}}=\lim _{r} Z_{p^{r}}$ induced by the inclusion $Z_{p^{r}} \rightarrow Z_{p^{r+1}}$. Let $G$ be a finitely-generated odd torsion group so that $G=\bigoplus_{i=1}^{m} Z_{p_{i}^{\alpha_{i}}}$. Let $P=\left\{p_{1}, \ldots, p_{m}\right\}$ and $G_{k+1}=\oplus_{i=1}^{m} Z_{p_{i}^{\alpha_{i}+k}}$. Then $Q / Z_{P}$ $\cong \lim _{k} G_{k}$.

Since $\pi_{2 n+1}\left(M\left(G_{r}, 2 n\right)\right)=G_{r} \otimes Z_{2}=0$, and $M\left(G_{r}, 2 n\right)$ can be thought of as the $(2 n+1)$-skeleton of $K\left(G_{r}, 2 n\right)$, the obstructions to extending

$$
S^{2 n} \rightarrow K\left(G_{r}, 2 n\right)^{(2 n+1)}
$$

to $\Omega S^{2 n+1} \rightarrow K\left(G_{r}, 2 n\right)^{(2 n+1)}$ are the obstructions to extending

$$
S^{2 n} \rightarrow M\left(G_{r}, 2 n\right)
$$

to $\Omega S^{2 n+1} \rightarrow M\left(G_{r}, 2 n\right)$.

Let $j_{r, m}: M\left(G_{r}, 2 n\right) \rightarrow M\left(G_{r+m}, 2 n\right)$ be inclusion and let $j: S^{2 n} \rightarrow M\left(G_{r}, 2 n\right)$.

THEOREM 6. For every $k$ and $r$, there is an $m$ such that $j_{r, m} \cdot j$ extends to $\left(S^{2 n}\right)_{k}$, the kth reduced product of $S^{2 n}$.

Proof. Let $j_{r, \infty}: M\left(G_{r}, 2 n\right) \rightarrow M\left(Q / Z_{P}, 2 n\right)$ be the inclusion. Since $M\left(Q / Z_{P}, 2 n\right)$ is an $H$-space, it follows that $j_{r, \infty} \cdot j$ extends to $\left(S^{2 n}\right)_{k}$ for all $k$. Let $\bar{j}$ be such an extension. Since $\left(S^{2 n}\right)_{k}$ is compact, image $\bar{j} \subseteq M\left(G_{r+m}, 2 n\right)$ for some $m$ and, hence, $\bar{j}$ is an extension of $j_{r, m} \cdot j$. 


\section{BIBLIOGRAPHY}

1. J. F. Adams, The sphere, considered as an $H$-space $\bmod p$, Quart J. Math. Oxford Ser. (2) 12 (1961), 52-60. MR 23 \# A651.

2. R. D. Porter, An H-space with finite dimensional homology whose loop space has torsion, Proc. Amer. Math. Soc. 37 (1973), 291-292. MR 46 \#9970.

3. J. D. Stasheff, On extensions of $H$-spaces, Trans. Amer. Math. Soc. 105 (1962), 126-135. MR 31 \#2726.

4. S. Weingram, On the incompressibility of certain maps, Ann. of Math. (2) 93 (1971), 476-485. MR $46 \# 890$.

Department of Mathematics, University of Wisconsin, MilwaUkeE, Wisconsin 53201 